\title{
Problems of Quantum Theory may be Solved by an Emulation Theory of Quantum Physics
}

\author{
Richard Woesler \\ German Aerospace Center, Institute of Transport Research, Rutherfordstr. 2, 12489 Berlin, Germany, \\ richard.woesler@dlr.de
}

\begin{abstract}
The emulation interpretation of quantum theory is described which may solve problems of the Copenhagen interpretation finally. According to Kolmogorov complexity theory it is conceivable that a bit string exists encoding our world which can be computed by an appropriate generalized Turing machine. In this case the computation would emulate the world, therefore this can be called an emulation theory of quantum physics, and the emulation interpretation of quantum theory. The probability of a string is dominated by the probabilities of its shortest programs which is known as the 'coding theorem'. This leads to the suggestion that there may be a relatively short shortest program by which our world may be run. This suggestion appears to be in accordance with our world. The world exhibits a number of symmetries. It is plausible that the shortest algorithm for our special world is shorter than those for worlds where symmetries are broken more often than in our world, because each further deviation from a symmetry has to be encoded within the algorithm which would enlarge its length. Therefore, laws of physics may be identical rather globally in spacetime. Further, in the Copenhagen interpretation of quantum theory it is defined, how to compute probabilities for, e.g., measurement results when conducting measurements on variables of quantum systems. In a completely satisfactory theory of everything this would not be sufficient, but such a theory should give a reason why the values of the probabilities seem, as far as it is known, to be identical also in all different regions of the observed world. The emulation interpretation suggests that all deviations from this symmetry of the probabilities would enlarge the shortest program of the world, and, therefore, we would probably not live in a world with such deviations. A second question arises from the attempt to combine the theory of black holes, thermodynamics and quantum theory. Bekenstein derives a holography principle which would restrict the number of degrees of freedom that can be present within a bounding surface to a finite number. In case the principle holds, he suggests that the final theory may be a discrete theory. The emulation interpretation is discrete. A promising detailed discrete theory which is currently developed is loop quantum gravity. Its discreteness was derived from some mathematical principles. It is also conceivable that string theories and/or M-theory can be unified with loop quantum gravity in future to a discrete theory. Additionally, the emulation interpretation suggests that parameters of physics may be encoded by a finite number of bits, they may be rational numbers, events in quantum physics may not be random but in principle computable, and, in a certain sense, space and time may be discrete variables. Falsifiability of the results is discussed.
\end{abstract}

\section{INTRODUCTION}

It is conceivable that there is a mathematical structure that describes our universe [1-3]. However, there may be limitations to compute physics as has been pointed out by Geroch and Hartle [4].

Within this article we use the hypothesis that our universe can be described by a mathematical structure. According to quantum theory, e.g., when a measurement is conducted on certain quantum systems, different results can occur with different probabilities. It is possible to interpret this in the following way. There are many possible worlds in which all possible measurement results occur, and we live in one of these worlds. Within this article we use the term world in this sense and the term universe for all these many worlds taken together.

Schmidhuber analysed the conceivable case that there is a bit string encoding our world which can be computed by a theoretical computer program using infinite storage, i.e. by an appropriate generalised Turing machine [1-2]. Applying Kolmogorov complexity theory leads to the suggestion that it may well be that our world can be computed by a program of finite code length. Additionally, the probability of a string is dominated by the probabilities of its 
shortest programs, which is known as the 'coding theorem' [1]. Therefore, this suggests that there is a relatively short shortest code, including the code for the definition of the Turing machine, by which we may be run [1-2].

This suggestion appears to be in nice accordance with the knowledge about our world. The world exhibits a number of symmetries. Symmetries are important in physical theories. The question why symmetries hold with high accuracy may be finally answered by the information theoretic approach. It is plausible that the shortest algorithm for our special world is shorter than those for worlds where symmetries are broken more often, because each deviation from a symmetry has to be encoded within the algorithm which enlarges the length of the code. We would probably live in a special world which has got a short shortest algorithm, and therefore, in a world with high degree of symmetries in physics.

The laws of physics may be the same here and on neighbouring planets, and rather globally in spacetime, because this corresponds to a short shortest code for the laws of physics of our world.

In the following text, problems of the Copenhagen interpretation of quantum theory are described, afterwards the emulation interpretation is described. Then, the question whether values of parameters of physics, in case such parameters exist, are restricted to certain possible values is considered. Afterwards, the falsifiability of the results is investigated. Finally, the results are discussed.

\section{TWO PPROBLEMS, OR QUESTIONS, IN THE COPENHAGEN INTERPRETATION OF QUANTUM THEORY}

In the Copenhagen interpretation of quantum theory it is defined how to compute probabilities from wave functions for, e.g., measurement results when conducting measurements on variables of quantum systems. In a completely satisfactory theory of everything it would not be sufficient just to define, how to compute these probabilities from functions. Such a theory should be able to give a reason why the values of the probabilities seem, as far as we know, to be identical also in all different regions of the observed world.

A second question arises from the attempt to combine the theory of black holes, thermodynamics and quantum theory. Bekenstein derives a holography principle which restricts the number of degrees of freedom that can be present within a bounding surface to a finite number [5]. In case the principle holds, he concludes that continuous field theory and superstring theories which have an infinity of degrees of freedom may not be the final theories. Instead, he thinks of a discrete theory where rather information exchange among physical processes shall be described. A discrete theory which is currently developed is loop quantum gravity [6,7]. The discreteness of the theory has been derived from mathematical principles. Bekenstein predicted that the entropy of a black hole is proportional to the area of its event horizon. The same result has also been found for loop quantum gravity [7].

An important alternative theory, M-theory, which is a unification of five detailed string theories and 11D super gravity, predicts 7 extra space dimensions which are usually supposed to be curled up into small spaces. From this new forces are predicted by the theory. However, down to about $100 \mu \mathrm{m}$ no new forces down to about gravitational strength have been found [8]. Also M-theory may be unified in future with loop quantum gravity towards a discrete theory [9].

\section{THE EMULATION INTERPRETATION OF QUANTUM PHYSICS}

As explained above, information theoretic arguments suggest that our special world may be run on a theoretical computer, e.g. on an appropriate generalised Turing machine, by a short algorithm [1-2]. In case this is true, the world can be simulated exactly by a program. An exact simulation is an emulation. This motivates an emulation interpretation of quantum physics. Rewriting quantum theory in terms of a computer simulation, e.g. as it is done when simulating quantum theory on a computer, may be called a simulation theory of quantum physics. An exact simulation of physics would constitute an emulation theory of quantum physics, and a hypothesis for such an exact simulation may also be called 'emulation theory of quantum physics'.

Consider the question why the values of the probabilities in quantum physics seem to be identical in the entire world, e.g. also in neighbouring star systems. In the traditional interpretation of quantum physics this is defined to be the case, but of course, the theory gives no reason for it. It seems that there may be much more possible worlds, in which the probabilities would differ, e.g., in the neighbouring star systems. In such other worlds in each star system the quantum probabilities within the same kind of experiments would show arbitrary different values. The information theoretic approach gives the hint, that short algorithms have, in a certain sense, higher probabilities [1- 
2]. It seems plausible that the shortest algorithm, in which probabilities for quantum processes are defined to differ in different regions of the observed world, consists of more bits than that of our world, because all differences have to be encoded within the code. Therefore, this may provide the final explanation for the identity of the probability distributions for quantum processes in the whole observed world.

I.e. the emulation interpretation may solve this problem of the Copenhagen interpretation of quantum physics. Furthermore, the emulation interpretation is a discrete theory, and discreteness is in accordance with the theoretical results of Bekenstein and of loop quantum gravity as mentioned above [5-7].

\section{CONSEQUENCES FOR PHYSICS AND FOR PARAMETERS OF PHYSICS}

In case the emulation interpretation of quantum theory is true, physics has got the following properties. Our world may be run by a theoretical computer program of finite code length. Parameters of physics, whether constant or not, may be encoded by a finite number of bits, they may be rational numbers. In a sense space and time may be discrete variables. Finally, all results of measurements observed in quantum physics may not be random but in principle even computable, because true randomness would not be possible using a finite input program only [1-2].

We remark that Tegmark proposed as theory of everything the ultimate ensemble theory of mathematical structures [3]. He considered large departures from our world which often lead to worlds within which self-aware structures cannot evolve, therefore, we do not live in such worlds. He stated that many obvious small departures from our island of habitability remain to be better explored in his framework, for instance: changing the spacetime topology, either on cosmological scales or on microscopic scales, adding and removing low-mass particles (fields) etc.

Complexity theory gives a hint that small, e.g., local departures would enlarge the code of our world, and therefore, we may not live in a world with such small departures.

\section{FALSIFIABILITY OF THE RESULTS}

The consequences of the emulation interpretation of quantum physics stated above may be supported by future experiments when such experiments suggest that dimensionless parameters in physics have got rational values, or that a deviation from true randomness is observed.

For the discrete loop quantum gravity predictions have been made which may be tested within the next years [7]. One prediction is that extremely high energy photons should have slightly different velocities. This may be testable with the planned Gamma ray Large Area Space Telescope (GLAST). Preliminary modifications to special relativity to allow for such different velocities of photons have been proposed.

Additionally, cosmic rays with energies above $10^{19}$ electron volts have been detected by the Akeno Giant Air Shower Array (AGASA). Usually it is assumed that cosmic protons of such high energies cannot reach earth. However, loop quantum gravity may cause that such protons can reach earth. In case the rays detected by AGASA were caused by protons, they may be a hint for loop quantum gravity theory [7].

\section{DISCUSSION}

The non-trivial information theoretic approach leads to the suggestion that it may be probable that parameters of physics, which may vary in space and time, may be rational numbers, that no true randomness may be present, and that in a certain sense time and space may be discrete variables.

As described in the present text, the emulation interpretation of quantum physics based on the information theoretic approach may yield the final explanations for the astonishing fact that laws of physics may be the same on earth as in neighbouring star systems, and for the identity of the values of the probabilities observed within quantum physics in different regions of the world. Further, the discreteness of the emulation interpretation would be in accordance with the result of Bekenstein and with loop quantum gravity as described above [5-7].

The analysis of generalized Turing machines [2] may be further generalized to explicitly include rather arbitrary Turing machines parallelly, and finally to explicitly include more and more mathematical structures. However, the result [1-2] that worlds with short shortest programs may have higher probabilities may indicate that rather simple 
generalized Turing machines, which can be described by short codes, like those analyzed so far may already yield results of great value.

Further, complexity analysis and the emulation interpretation provide an approach to a theory of physics. Theories like loop quantum gravity could be formulated in terms of computer programs. In future small rather random modifications can be made to such programs automatically, and the modified programs can be tested and evaluated which could eventually yield contributions to a final theory of physics.

\section{REFERENCES}

1. Schmidhuber, J., Foundations of Computer Science: Potential-Theory-Cognition, Lecture Notes in Computer Science 1337, 201-208D (1997).

2. Schmidhuber, J., International Journal of Foundations of Computer Science 13 (4), 587-612 (2002).

3. Tegmark, M., Annals of Physics 270, 1-51R (1998).

4. Geroch, R., and Hartle, J. B., Foundations of Physics 16 (6), 533-550W (1986).

5. Bekenstein, J. D., Scientific American, 289 (2), 58-65 (2003).

6. Baez, J., Nature 421, $702-703$ (2003).

7. Smolin, L., Scientific American, 290 (1), 66-75 (2004).

8. Long, J. C., et al., Letters to Nature, Nature 421, 922-925 (2003).

9. Scientific American, 289 (5), 68-73 (2003). 